\title{
Fuzzy Observer-based Robust Guaranteed Cost Fault-tolerant Design for Uncertain Nonlinear NCS
}

\author{
Jun Wang ${ }^{1, *}$, Wei Li $^{1}$ and Ling Guan ${ }^{2}$ \\ ${ }^{1}$ College of Electrical and Information Engineering, Lanzhou University of Technology, Lanzhou, 730050, China \\ ${ }^{2}$ GNGC Gansu Yinguang Chemicals Industria l(G) Co., Ltd. Energetic material Fellow Subsidiary, Baiyin, 730900, \\ China
}

\begin{abstract}
Through the comparison and analysis of some recent patents on the fault-tolerant designed method, this paper addresses the problem of robust guaranteed cost fault-tolerant for nonlinear networked control systems (NNCS) with network-induced delay and packed dropout. Firstly, we adopt observer and state-feedback control strategy, wherein states are immeasurable. Then, by constructing appropriate Lyapunov-Krasovskii functional, based on T-S fuzzy model, a delaydependent sufficient condition is deduced for robust guaranteed cost fault-tolerant control. Further, observer-based gain and state-feedback controller gain can be obtained and optimized. Finally, an example is used to illustrate the effectiveness and feasibility of the proposed approach.
\end{abstract}

Keywords: Fault-tolerant control, Guaranteed cost, Nonlinear Networked Control System, state observer, T-S Fuzzy Model.

\section{INTRODUCTION}

According to European Patent 2075961 [1] and U.S. Patent 7426189 [2], (Networked Control System) NCS is a control system of full-distributed real-time feedback which connects the sensors, controllers and actuators from different locations through network.

In the past decade, NCS has become a widespread concern in research areas. Although NCS has advantages of a low cost, easy installation and maintenance, system reliability and flexibility, easy to fault diagnosis, etc., network delay and packet dropout have become primary problems, which cannot be avoided due to bandwidth, irregular flow and unreliable transmission. Not only does it cause the system performance degradation, and even lead to system instability [35]. In addition, compared with traditional systems, NCS is larger in scale and more complicated in structure, therefore all kinds of uncertainty and fault-induced factors have greatly increased. As a result more and more researchers have started to focus on the fault-tolerant control of NCS [68], hoping to get better performances and higher safety and reliability $[9,10]$.

In the practical, the nonlinear properties more or less exist in NCS. Due to its complexity, there are fewer studies on the fault tolerant. The preceding fault-tolerant control researches on NNCS are discussed by adopting state-feedback control strategies [11]. However, not all states can be

*Address correspondence to this author at the College of Electrical and information engineering,Lanzhou University of Technology, Lanzhou, 730050, China; Tel: 0931-2976020; Fax: 0931-2973506;

E-mail: wangj31901@163.com measured. Therefore, targeted at a class of uncertain NNCS and based on observer of fuzzy state, this paper studies the control problem of the robust fault-tolerant guaranteed cost while considering the influences of time-delay and packet dropout under the condition of actuator failures.

\section{SYSTEM DESCRIPTION}

Typical uncertain NNCS described by T-S fuzzy model is shown in Fig. (1):

In Fig. (1), $\tau_{c a}$ and $!_{s c}$ are the time-varying networkinduced delays from controller to actuator and from sensor to controller, respectively.

Assumption 1: It is assumed that the sensor is clockdriven, while the controller and actuator are event-driven.

Assumption 2: The sampled data is transmitted with a single packet. The packets reach the controller and actuator by their original transmitting sequence if they are not lost.

Assumption 3: Data packet dropouts are used as a special kind of time delays.

Therefore, we can get according to $[12,13]$

$$
\begin{aligned}
& \tau_{s c 1} \leq \tau_{1}(t) \leq\left(\delta_{1 \mathrm{M}}+1\right) T+\tau_{s c 2} \\
& \tau_{c a 1} \leq \tau_{2}(t) \leq\left(\delta_{2 \mathrm{M}}+1\right) T+\tau_{c a 2} \\
& \text { Define } \tau_{s 2}=\left(\delta_{1 \mathrm{M}}+1\right) T+\tau_{s c 2}, \tau_{s 1}=\tau_{s c 1} \\
& \text { Then } \tau_{s 1} \leq \tau_{1}(t) \leq \tau_{s 2}, \\
& \text { similarly have } \tau_{c 1} \leq \tau_{2}(t) \leq \tau_{c 2}
\end{aligned}
$$




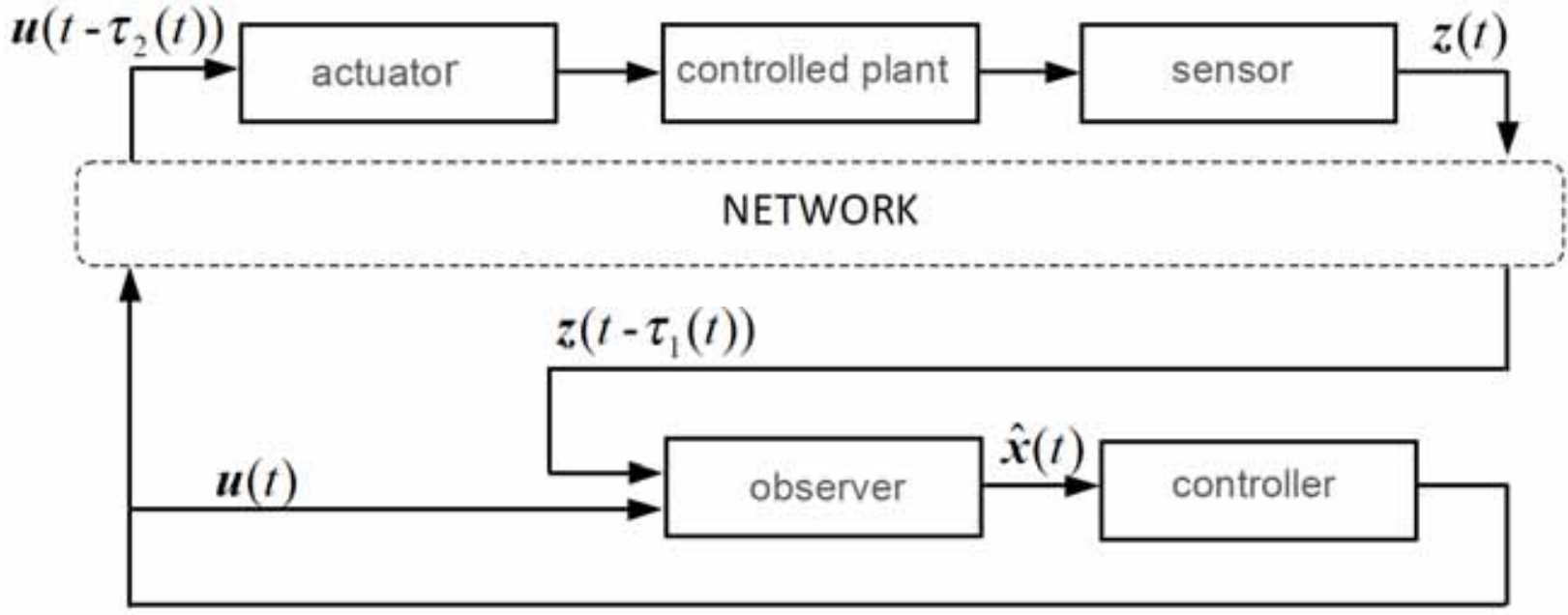

Fig. (1). Framework of networked control systems Based on state observer.

where $\tau_{1}(t)$ and $\tau_{2}(t)$ are total time-varying delays respectively of controller-to-actuator and sensor-to- controller including network-induced delays and data packet dropouts. $\tau_{s c 1}, \tau_{s c 2}$ and $\tau_{c a 1}, \tau_{c a 2}$ are positive constant representing the lower and upper delay bound of corresponding transmission channel, respectively. $\delta_{1 M}$ and $\delta_{2 M}$ are the maximum number of data packet dropouts of corresponding transmission channel, respectively.

According to Japanese Patent JP 06035508 [14], consider a NNCS with parameter uncertainties represented by T-S fuzzy model as follows:

Fuzzy rule i: if $\theta_{1}(\mathrm{t})$ is $\mathrm{F}_{\mathrm{i} 1}$ and if $\theta_{2}(\mathrm{t})$ is $\mathrm{F}_{\mathrm{i} 2}$ and $\ldots$ and $\theta_{\mathrm{n}}(\mathrm{t})$ is Fin, then

$$
\begin{aligned}
& \dot{\boldsymbol{x}}(t)=\left(\boldsymbol{A}_{i}+\Delta \boldsymbol{A}_{i}\right) \boldsymbol{x}(t)+\left(\boldsymbol{B}_{i}+\Delta \boldsymbol{B}_{i}\right) \boldsymbol{u}\left(t-\tau_{2}(t)\right) \\
& z(t)=C_{i} \boldsymbol{x}\left(t-\tau_{1}(t)\right) \quad(i=1,2, \ldots, N)
\end{aligned}
$$

where $\mathrm{i}$ is the number of if-then rules; $F_{i j}(j=1,2, \ldots, n)$ and $\theta(\mathrm{t})=\left[\theta_{1}(\mathrm{t}), \theta_{2}(\mathrm{t}), \ldots, \theta_{\mathrm{n}}(\mathrm{t})\right] \mathrm{T}$ are fuzzy sets and premise variables, respectively; $\boldsymbol{x}(t) \in R^{n}, \boldsymbol{u}(t) \in R^{m}$ and $z(t) \in R^{l}$ are state vector, control input vector and output control vector, respectively; $\boldsymbol{A}_{i} \boldsymbol{B}_{i}$ and $\boldsymbol{C}_{i}$ are constant matrices with appropriate dimensions, respectively; $\Delta \boldsymbol{A}_{i}$ and $\Delta \boldsymbol{B}_{i}$ are time-varying unknown matrices with appropriate dimensions, respectively, which stand for uncertainty of structure in the system model and can be described as

$\left[\Delta \boldsymbol{A}_{i}, \quad \Delta \boldsymbol{B}_{i}\right]=\boldsymbol{D F}(t)\left[\boldsymbol{E}_{a i}, \quad \boldsymbol{E}_{b i}\right]$

where $\boldsymbol{D} 、 \boldsymbol{E}_{a i}$ and $\boldsymbol{E}_{b i}$ are known constant matrices with appropriate dimensions; $\boldsymbol{F}(t)$ is an unknown matrix function with Lebesgue measurable elements satisfying the inequality $\boldsymbol{F}^{T}(t) \boldsymbol{F}(t) \leq \boldsymbol{I}$.

For any given $\boldsymbol{x}(t)$ and $\boldsymbol{u}(t)$, by using a singleton fuzzifier, product inference and centre-average defuzzifier, the local models can be integrated into a global nonlinear model:

$$
\begin{aligned}
& \dot{\boldsymbol{x}}(t)=\sum_{i=1}^{N} \mu_{i}(\theta(t))\left[\left(\boldsymbol{A}_{i}+\Delta \boldsymbol{A}_{i}\right) \boldsymbol{x}(t)+\left(\boldsymbol{B}_{i}+\Delta \boldsymbol{B}_{i}\right) \boldsymbol{u}\left(t-\tau_{2}(t)\right)\right] \\
& \boldsymbol{z}(t)=\sum_{i=1}^{N} \mu_{i}(\theta(t)) \boldsymbol{C}_{i} \boldsymbol{x}\left(t-\tau_{1}(t)\right)
\end{aligned}
$$

where $\mu_{i}(\theta(t))=\frac{a_{i}(\theta(t))}{\sum_{i=1}^{N} a_{i}(\theta(t))}$, and $\mu_{i}(\theta(t))$ is the weight ratio of each fuzzy rule satisfying $\mu_{i}(\theta(t)) \geq 0 \quad(i=1,2, \ldots, N)$ and $\sum_{i=1}^{N} \mu_{i}(\theta(t))=1 ; a_{i}(\theta(t))=\prod_{j=1}^{n} F_{i j}\left(\theta_{j}(t)\right)$, and $F_{i j}\left(\theta_{j}(t)\right)$ is grade of the membership of $\theta \mathrm{j}(\mathrm{t})$ in fuzzy set Fij, and it is assumed that $a_{i}(\theta(t)) \geq 0(i=1,2, \ldots, N)$ and $\sum_{i=1}^{N} a_{i}(\theta(t))>0$.

It is assumed that all of the state variables are unmeasurable for NNCS, but it is observable. According to parallel distributed compensation (PDC) technique, the fuzzy dynamic output feedback controller is observer-based of fulldimension which shares the same premise parts as the fuzzy system, and has the following form

$$
\begin{gathered}
\dot{\hat{\boldsymbol{x}}}(t)=\sum_{i=1}^{N} \mu_{i}(\theta(t))\left[\left(\boldsymbol{A}_{i}+\Delta \boldsymbol{A}_{i}\right) \hat{\boldsymbol{x}}(t)+\left(\boldsymbol{B}_{i}+\Delta \boldsymbol{B}_{i}\right) \boldsymbol{u}(t)+\right. \\
\boldsymbol{I}_{i}\left(\boldsymbol{C}_{i} \boldsymbol{x}\left(t-\tau_{1}(t)\right)-\boldsymbol{C}_{i} \hat{\boldsymbol{x}}(t)\right] \\
\boldsymbol{u}(t)=\sum_{i=1}^{N} \mu_{i}(\theta(t)) \boldsymbol{K}_{i} \hat{\boldsymbol{x}}(t)
\end{gathered}
$$

where $\hat{\boldsymbol{x}}(t) \in R^{n}$ is the estimation of state vector $\boldsymbol{x}(t) ; \boldsymbol{K}_{j}$ is the control gain matrix for the jth controller rule, $\boldsymbol{I}_{i}$ is the observer gain matrix for the ith observer rule.

Substituting (6) into (4) and (5) yields the close-loop NNCS as follow

$$
\begin{gathered}
\dot{\hat{\boldsymbol{x}}}(t)=\sum_{i=1}^{N} \sum_{j=1}^{N} \mu_{i}(\theta(t)) \mu_{j}(\theta(t))\left[\left(\tilde{\boldsymbol{A}}_{i}+\tilde{\boldsymbol{B}}_{i} \boldsymbol{K}_{j}-\right.\right. \\
\left.\boldsymbol{I}_{i} \boldsymbol{C}_{i}\right) \hat{\boldsymbol{x}}(t)+\boldsymbol{I}_{i} \boldsymbol{C}_{i} \boldsymbol{x}\left(t-\tau_{1}(t)\right]
\end{gathered}
$$




$$
\dot{\boldsymbol{x}}(t)=\sum_{i=1}^{N} \sum_{j=1}^{N} \mu_{i}(\theta(t)) \mu_{j}(\theta(t))\left[\tilde{\boldsymbol{A}}_{i} \boldsymbol{x}(t)+\tilde{\boldsymbol{B}}_{i} \boldsymbol{K}_{j} \hat{\boldsymbol{x}}\left(t-\tau_{2}(t)\right)\right]
$$

where $\tilde{\boldsymbol{A}}_{i}=\boldsymbol{A}_{i}+\Delta \boldsymbol{A} 、 \quad \tilde{\boldsymbol{B}}_{i}=\boldsymbol{B}_{i}+\Delta \boldsymbol{B}$

Defining the estimation error $\boldsymbol{e}(t)=\hat{\boldsymbol{x}}(t)-\boldsymbol{x}(t)$, we get

$$
\begin{aligned}
\dot{\boldsymbol{e}}(t)=\sum_{i=1}^{N} & \sum_{j=1}^{N} \mu_{i}(\theta(t)) \mu_{j}(\theta(t))\left[\left(\tilde{\boldsymbol{A}}_{i}+\tilde{\boldsymbol{B}}_{i} \boldsymbol{K}_{j}-\boldsymbol{I}_{i} \boldsymbol{C}_{i}\right) \boldsymbol{e}(t)+\right. \\
& \left(\tilde{\boldsymbol{B}}_{i} \boldsymbol{K}_{j}-\boldsymbol{I}_{i} \boldsymbol{C}_{i}\right) \boldsymbol{x}(t)+\boldsymbol{I}_{i} \boldsymbol{C}_{i} \boldsymbol{x}\left(t-\tau_{1}(t)\right)- \\
& \left.\tilde{\boldsymbol{B}}_{i} \boldsymbol{K}_{j} \boldsymbol{x}\left(t-\tau_{2}(t)\right)-\tilde{\boldsymbol{B}}_{i} \boldsymbol{K}_{j} \boldsymbol{e}\left(t-\tau_{2}(t)\right)\right]
\end{aligned}
$$

Lemma 1[15]: Given constant matrices $S, H$ and $E$ of appropriate dimensions and with matrix $\mathrm{S}$ symmetric, then

$$
\boldsymbol{S}+\boldsymbol{H} \boldsymbol{F}(t) \boldsymbol{E}+\boldsymbol{E}^{T} \boldsymbol{F}^{T}(t) \boldsymbol{H}^{T}<0
$$

for all $\mathrm{F}(\mathrm{t})$ satisfying $\boldsymbol{F}^{T}(t) \boldsymbol{F}(t) \leq \boldsymbol{I}$, if and only if there exists a scalar $\varepsilon>0$ such that

$\boldsymbol{S}+\boldsymbol{\varepsilon} \boldsymbol{H} \boldsymbol{H}^{T}+\varepsilon^{-1} \boldsymbol{E}^{T} \boldsymbol{E} \triangleleft 0$

Lemma 2 (matrix separation lemma [16]). If

$$
\left[\begin{array}{cccc}
a_{11} & a_{12} & a_{13} & a_{14} \\
& a_{22} & a_{23} & a_{24} \\
& & a_{33} & a_{34} \\
* & & & a_{44}
\end{array}\right]<0,\left[\begin{array}{lll}
b_{11} & b_{12} & b_{13} \\
& b_{22} & b_{23} \\
* & & b_{33}
\end{array}\right]<0,\left[\begin{array}{ll}
c_{11} & c_{12} \\
& c_{22}
\end{array}\right]<0,
$$

then

$$
\left[\begin{array}{ccccc}
a_{11} & a_{12} & a_{13} & a_{14} & 0 \\
& a_{22} & a_{23} & a_{24} & 0 \\
& & a_{33} & a_{34} & 0 \\
& * & & a_{44} & 0 \\
& & & & 0
\end{array}\right]+\left[\begin{array}{ccccc}
0 & 0 & 0 & 0 & 0 \\
& b_{11} & 0 & b_{12} & b_{13} \\
& & 0 & 0 & 0 \\
& & & b_{22} & b_{23} \\
& & & & b_{33}
\end{array}\right]+\left[\begin{array}{ccccc}
c_{11} & c_{12} & 0 & 0 & 0 \\
& c_{22} & 0 & 0 & 0 \\
& & 0 & 0 & 0 \\
& * & & 0 & 0 \\
& & & & 0
\end{array}\right]<0
$$

\section{MAIN RESULTS}

\subsection{Robust Guaranteed Cost Fault-tolerant Analysis of NNCS with Actuator Failures Fore-casting}

Considering the possible actuator failures, we can introduce a switching matrix $\boldsymbol{L}$ as follow

$$
\boldsymbol{L}=\operatorname{diag}\{l 1, l 2, \ldots \cdot \ln \},
$$

where $l_{i}= \begin{cases}1, & \text { the ith actuator normal } \\ 0, & \text { the ith actuator failure }\end{cases}$

$\boldsymbol{L} \in \Omega$, and $\Omega$ denotes the set of all possible actuator failures switching matrices except $\boldsymbol{L}=0$.

When the switching matrix $\boldsymbol{L}$ is laid between the feedback matrix $\boldsymbol{K}_{j}$ and input matrix $\tilde{\boldsymbol{B}}_{i}$, we get the following uncertain nonlinear networked closed-loop fault system (UNNCFS)

$$
\begin{aligned}
& \dot{\boldsymbol{x}}(t)= \sum_{i=1}^{N} \sum_{j=1}^{N} \mu_{i}(\theta(t)) \mu_{j}(\theta(t))\left[\tilde{\boldsymbol{A}}_{i} \boldsymbol{x}(t)+\right. \\
&\left.\tilde{\boldsymbol{B}}_{i} \boldsymbol{L} \boldsymbol{K}_{j} \boldsymbol{x}\left(t-\tau_{2}(t)\right)+\tilde{\boldsymbol{B}}_{i} \boldsymbol{L} \boldsymbol{K}_{j} \boldsymbol{e}\left(t-\tau_{2}(t)\right)\right]
\end{aligned}
$$

$$
\begin{aligned}
\dot{\boldsymbol{e}}(t)= & \sum_{i=1}^{N} \sum_{j=1}^{N} \mu_{i}(\theta(t)) \mu_{j}(\theta(t))\left[\left(\tilde{\boldsymbol{A}}_{i}+\tilde{\boldsymbol{B}}_{i} \boldsymbol{L} \boldsymbol{K}_{j}-\boldsymbol{I}_{i} \boldsymbol{C}_{i}\right) \boldsymbol{e}(t)+\right. \\
& \left(\tilde{\boldsymbol{B}}_{i} \boldsymbol{L} \boldsymbol{K}_{j}-\boldsymbol{I}_{i} \boldsymbol{C}_{i}\right) \boldsymbol{x}(t)+\boldsymbol{I}_{i} \boldsymbol{C}_{i} \boldsymbol{x}\left(t-\tau_{1}(t)\right)- \\
& \tilde{\boldsymbol{B}}_{i} \boldsymbol{L} \boldsymbol{K}_{j} \boldsymbol{x}\left(t-\tau_{2}(t)-\tilde{\boldsymbol{B}}_{i} \boldsymbol{L} \boldsymbol{K}_{j} \boldsymbol{e}\left(t-\tau_{2}(t)\right]\right.
\end{aligned}
$$

According to the UNNCFS, we define the guaranteed cost function

$J=\int_{0}^{\infty}\left[x^{T}(t) \boldsymbol{Q} x(t)+u^{T}(t) \boldsymbol{R} u(t)\right] d t$

where $\boldsymbol{Q}$ and $\boldsymbol{R}$ are given positive definite symmetric matrices.

In our study, based on observer, the aim of robust faulttolerant guaranteed cost control is to seek the control gain matrix $\boldsymbol{K}_{j}$ and the observer gain matrix $\boldsymbol{I}_{i}$ such that the UNNCFS (10)-(11) is asymptotically stable and the guaranteed cost function $J$ satisfies $J \leq J^{*}$, and then $J^{*}$ is said to upper boundary of guaranteed cost function.

Theorem 1: For the UNNCFS described by (10)-(11) and the guaranteed cost function $J$, given positive constants $\tau_{s c 1}, \tau_{s c 2}, \tau_{c a 1}, \tau_{c a 2}, \delta_{1 M}, \delta_{2 M}$ and $d$, if there exit matrices, $\overline{\boldsymbol{Q}}=\overline{\boldsymbol{Q}}^{T}>0, \boldsymbol{R}=\boldsymbol{R}^{T}>0, \overline{\boldsymbol{R}}_{i}=\overline{\boldsymbol{R}}_{i}^{T}>0, \overline{\boldsymbol{Z}}_{i}=\overline{\boldsymbol{Z}}_{i}^{T}>0 \quad(i=1,2)$, $\overline{\boldsymbol{Q}}_{i j}=\overline{\boldsymbol{Q}}_{i j}^{T}>0 \quad(i=1,2, \mathrm{j}=1,2)$, , and $\boldsymbol{X}, \boldsymbol{Y}_{1 j}, \boldsymbol{Y}_{2 i} \quad(i, j=1,2, \ldots, N)$ with appropriate dimensions, satisfying the following LMIs

$$
\left[\begin{array}{ccccc}
\overline{\boldsymbol{D}}_{i j}^{1} & \overline{\boldsymbol{H}}_{1} & \varepsilon_{1}^{-1} \overline{\boldsymbol{E}}_{1}^{T} & \overline{\boldsymbol{H}}_{2} & \varepsilon_{2}^{-1} \overline{\boldsymbol{E}}_{2}^{T} \\
& -\varepsilon_{1}^{-1} \boldsymbol{I} & 0 & 0 & 0 \\
& & -\varepsilon_{1}^{-1} \boldsymbol{I} & 0 & 0 \\
& * & & -\varepsilon_{2}^{-1} \boldsymbol{I} & 0 \\
& & & & -\varepsilon_{2}^{-1} \boldsymbol{I}
\end{array}\right]<0
$$

$$
\left[\begin{array}{ccccc}
0 & 0 & -\boldsymbol{C}_{i}^{T} \boldsymbol{Y}_{2 i}^{T} & -\boldsymbol{C}_{i}^{T} \boldsymbol{Y}_{2 i}^{T} \tau_{c 2} & -\boldsymbol{C}_{i}^{T} \boldsymbol{Y}_{2 i}^{T} \tau_{s 2} \\
& 0 & \boldsymbol{C}_{i}^{T} \boldsymbol{Y}_{2 i}^{T} & \boldsymbol{C}_{i}^{T} \boldsymbol{Y}_{2 i}^{T} \tau_{c 2} & \boldsymbol{C}_{i}^{T} \boldsymbol{Y}_{2 i}^{T} \tau_{s 2} \\
& -\boldsymbol{Y}_{2 i} \boldsymbol{C}_{i}- & & \boldsymbol{C}_{i}^{T} \boldsymbol{Y}_{2 i}^{T} \tau_{c 2} & -\boldsymbol{C}_{i}^{T} \boldsymbol{Y}_{2 i}^{T} \tau_{s 2} \\
& \boldsymbol{C}_{i}^{T} \boldsymbol{Y}_{2 i}^{T} & 0 & 0 \\
* & & 0 & 0
\end{array}\right]<0
$$

Where

$$
\begin{aligned}
\overline{\boldsymbol{D}}_{i j}^{1} & =\left[\begin{array}{ccccc}
\overline{\boldsymbol{D}}_{1 i j}^{1} & \overline{\boldsymbol{D}}_{2 i j}^{1} \\
* & \boldsymbol{D}_{3 i j}^{1}
\end{array}\right]<0 \\
\overline{\boldsymbol{D}}_{1 i j}^{1}= & {\left[\begin{array}{cccccc}
\overline{\boldsymbol{O}}_{11} & \overline{\boldsymbol{R}}_{1} & \overline{\boldsymbol{O}}_{13} & \overline{\boldsymbol{O}}_{14} & 0 & \overline{\boldsymbol{O}}_{16} \\
& -\overline{\boldsymbol{R}}_{1} & 0 & 0 & 0 & 0 \\
& & -\overline{\boldsymbol{Z}}_{1} & \overline{\boldsymbol{O}}_{34} & 0 & 0 \\
& & & \overline{\boldsymbol{O}}_{44} & \overline{\boldsymbol{R}}_{2} & \overline{\boldsymbol{O}}_{46} \\
& * & & & -\overline{\boldsymbol{R}}_{2} & 0 \\
& & & & & -\overline{\boldsymbol{Z}}_{2}
\end{array}\right] }
\end{aligned}
$$


$\overline{\boldsymbol{O}}_{11}=\boldsymbol{A}_{i} \boldsymbol{X}^{T}+\boldsymbol{X} \boldsymbol{A}_{i}^{T}+\overline{\boldsymbol{Q}}_{11}+\overline{\boldsymbol{Q}}_{21}-\overline{\boldsymbol{R}}_{1}-\overline{\boldsymbol{Z}}_{1}+\overline{\boldsymbol{Q}}$

$\overline{\boldsymbol{O}}_{13}=\boldsymbol{B}_{i} \boldsymbol{L} \boldsymbol{Y}_{1 j}+\overline{\boldsymbol{Z}}_{1} \overline{\boldsymbol{O}}_{14}=d \boldsymbol{Y}_{1 j}^{T} \boldsymbol{L}^{T} \boldsymbol{B}_{i}^{T}$

$\ddot{\boldsymbol{O}}_{16}=\boldsymbol{B}_{i} \boldsymbol{L} \boldsymbol{Y}_{1 j} \quad \ddot{\boldsymbol{O}}_{34}=-d \boldsymbol{Y}_{1 j}^{T} \boldsymbol{L}^{T} \boldsymbol{B}_{i}^{T}$

$\ddot{\boldsymbol{O}}_{44}=d \boldsymbol{A}_{i} \boldsymbol{X}^{T}+d \boldsymbol{X} \boldsymbol{A}_{i}^{T}+d \boldsymbol{B}_{i} \boldsymbol{L} \boldsymbol{Y}_{1 j}+$

$$
d \boldsymbol{Y}_{1 j}^{T} \boldsymbol{L}^{T} \boldsymbol{B}_{i}^{T}-\overline{\boldsymbol{R}}_{2}-\overline{\boldsymbol{Z}}_{2}+\overline{\boldsymbol{Q}}_{12}+\overline{\boldsymbol{Q}}_{22}
$$

$\overline{\boldsymbol{O}}_{46}=-d \boldsymbol{B}_{i} \boldsymbol{L} \boldsymbol{Y}_{1 j}+\overline{\boldsymbol{Z}}_{2}$

$\overline{\boldsymbol{D}}_{2 i j}^{1}=\left[\begin{array}{ccccc}\boldsymbol{X} \boldsymbol{A}_{i}^{T} \tau_{c 2} & \boldsymbol{X} \boldsymbol{A}_{i}^{T} \tau_{s 2} & \ddot{\boldsymbol{O}}^{T} \tau_{c 2} & \ddot{\boldsymbol{O}}^{T} \tau_{s 2} & \boldsymbol{Y}_{j}^{T} \\ 0 & 0 & 0 & 0 & 0 \\ \overline{\boldsymbol{O}}^{T} \tau_{c 2} & \overline{\boldsymbol{O}}^{T} \tau_{s 2} & -\overline{\boldsymbol{O}}^{T} \tau_{c 2} & -\overline{\boldsymbol{O}}^{T} \tau_{s 2} & 0 \\ 0 & 0 & \left(\boldsymbol{X} \boldsymbol{A}_{i}^{T}+\widetilde{\boldsymbol{O}}^{T}\right) \tau_{c 2} & \left(\boldsymbol{X} \boldsymbol{A}_{i}^{T}+\tilde{\boldsymbol{O}}^{T}\right) \tau_{s 2} & \boldsymbol{Y}_{j}^{T} \\ 0 & 0 & 0 & 0 & 0 \\ \ddot{\boldsymbol{O}}^{T} \tau_{c 2} & \ddot{\boldsymbol{O}}^{T} \tau_{s 2} & -\ddot{\boldsymbol{O}}^{T} \tau_{c 2} & -\ddot{\boldsymbol{O}}^{T} \tau_{s 2} & 0\end{array}\right]$

$\ddot{\boldsymbol{O}}=\boldsymbol{B}_{i} \boldsymbol{L} \boldsymbol{Y}_{1 j}$

$\boldsymbol{D}_{3 i j}^{1}=\operatorname{diag}\left[\begin{array}{lllll}0 & 0 & 0 & 0 & -\boldsymbol{R}^{-1}\end{array}\right]$

$\overline{\boldsymbol{H}}_{1}^{T}=\left[\begin{array}{lllllllll}\boldsymbol{D}^{T} & 0 & \cdots & 0 & \tau_{c 2} \boldsymbol{D}^{T} & \tau_{s 2} \boldsymbol{D}^{T} & 0 & 0 & 0\end{array}\right]_{1 \times 11}$

$\overline{\boldsymbol{E}}_{1}=\left[\begin{array}{llllllllll}\boldsymbol{E}_{a i} \boldsymbol{X}^{T} & 0 & \boldsymbol{E}_{b i} \boldsymbol{L} \boldsymbol{Y}_{1 j} & 0 & 0 & \boldsymbol{E}_{b i} \boldsymbol{L} \boldsymbol{Y}_{1 j} & 0 & \cdots & 0\end{array}\right]_{1 \times 11}$

$\overline{\boldsymbol{H}}_{2}^{T}=\left[\begin{array}{lllllllll}0 & 0 & 0 & d \boldsymbol{D}^{T} & 0 \cdots & 0 & \tau_{c 2} \boldsymbol{D}^{T} & \tau_{s 2} \boldsymbol{D}^{T} & 0\end{array}\right]_{1 \times 11}$

$\overline{\boldsymbol{E}}_{2}=\left[\begin{array}{llll}\boldsymbol{E}_{b i} \boldsymbol{L} \boldsymbol{Y}_{1 j} & 0 & -\boldsymbol{E}_{b i} \boldsymbol{L} \boldsymbol{Y}_{1 j} & \boldsymbol{E}_{a i} \boldsymbol{X}^{T}+\boldsymbol{E}_{b i} \boldsymbol{L} \boldsymbol{Y}_{1 j}\end{array}\right.$

$$
\begin{array}{lllll}
0 & -\boldsymbol{E}_{b i} \boldsymbol{L} \boldsymbol{Y}_{1 j} & 0 & \cdots & 0]_{\mid \times 11}
\end{array}
$$

then existing the observer-based state-feedback control law (5)-(6) for robust guaranteed cost fault-tolerant control such that UNNCFS(10)-(11) with $\boldsymbol{K}_{j}=\boldsymbol{Y}_{1 j} \boldsymbol{X}^{-T}$ and $\boldsymbol{I}_{i}=d^{-1} \boldsymbol{X} \boldsymbol{Y}_{2 i}$ is asymptotically stable, and the guaranteed cost function $J$ satisfies the following boundary.

$J \leq J^{*}=\left[\begin{array}{c}\boldsymbol{x}^{T}(0) \\ \boldsymbol{e}^{T}(0)\end{array}\right]^{T}\left[\begin{array}{cc}\boldsymbol{P}_{1} & 0 \\ 0 & \boldsymbol{P}_{2}\end{array}\right]\left[\begin{array}{l}\boldsymbol{x}(0) \\ \boldsymbol{e}(0)\end{array}\right]+$
$\sum_{i=1}^{2} \int_{-\alpha_{i}}^{0}\left[\begin{array}{c}\boldsymbol{x}^{T}(s) \\ \boldsymbol{e}^{T}(s)\end{array}\right]^{T}\left[\begin{array}{cc}\boldsymbol{Q}_{i 1} & 0 \\ 0 & \boldsymbol{Q}_{i 2}\end{array}\right]\left[\begin{array}{l}\boldsymbol{x}(s) \\ \boldsymbol{e}(s)\end{array}\right] d s+$

$\int_{-\tau_{c 2}}^{0} \tau_{c 2} \int_{\theta}^{0}\left[\begin{array}{c}\dot{\boldsymbol{x}}^{T}(s) \\ \dot{\boldsymbol{e}}^{T}(s)\end{array}\right]^{T}\left[\begin{array}{cc}\boldsymbol{R}_{1} & 0 \\ 0 & \boldsymbol{R}_{2}\end{array}\right]\left[\begin{array}{c}\dot{\boldsymbol{x}}(s) \\ \dot{\boldsymbol{e}}(s)\end{array}\right] d s d \theta+$

$\int_{-\tau_{s 2}}^{0} \tau_{s 2} \int_{\theta}^{0}\left[\begin{array}{c}\dot{\boldsymbol{x}}^{T}(s) \\ \dot{\boldsymbol{e}}^{T}(s)\end{array}\right]^{T}\left[\begin{array}{cc}\boldsymbol{Z}_{1} & 0 \\ 0 & \boldsymbol{Z}_{2}\end{array}\right]\left[\begin{array}{c}\dot{\boldsymbol{x}}(s) \\ \dot{\boldsymbol{e}}(s)\end{array}\right] d s d \theta$

Proof: the proof is cut off due to space limitation. contact the authors for the detailed proof.

Remark 1: In order to determine the observer gains $\boldsymbol{I}_{i}$, we have to set $\boldsymbol{P}_{2}=d \boldsymbol{P}_{1}$ to obtain the LMI conditions. The scalar $d$ should be given prior to solve LMI (13) and (14).
How to choose scalar $d$ for optimization is still an open problem. Hence, we determine the scalar $d$ by the trial and error method.

Remark 2: The theorem 1 provides a robust fault-tolerant guaranteed cost condition in form of delay-dependent, which include some of the information of delay from controller to actuator and from sensor to controller. Especially, introducing of lower delay bound, the result is less conservative [17].

\subsection{Optimization of Guaranteed Cost}

Control gain matrix $\boldsymbol{K}_{j}$ and observer gain matrix $\boldsymbol{I}_{i}$ can be solved via the above theorem 1 , and the guaranteed cost function $J$ satisfies $J \leq J^{*}$, but $J^{*}$ is not the minimum upper boundary of guaranteed cost, $\boldsymbol{K}_{j}$ and $\boldsymbol{I}_{i}$ gain matrices are only the suboptimal solution. Thence, we introduced the following optimization algorithm for optimal guaranteed cost gain matrices which minimize $J^{*}$.

$$
\begin{gathered}
\min J^{*}=\vartheta_{1}+\vartheta_{2}+\operatorname{Trace}\left(M_{11}\right)+\operatorname{Trace}\left(M_{12}\right)+ \\
\operatorname{Trace}\left(M_{21}\right)+\operatorname{Trace}\left(M_{22}\right)+\operatorname{Trace}\left(T_{1}\right)+ \\
\operatorname{Trace}\left(T_{2}\right)+\operatorname{Trace}\left(S_{1}\right)+\operatorname{Trace}\left(S_{2}\right)
\end{gathered}
$$

s.t. i) (13) ii) (14)

$$
\begin{aligned}
& \text { iii) }\left[\begin{array}{cc}
-\vartheta_{1} & \boldsymbol{x}^{T}(0) \\
* & -\boldsymbol{P}_{1}^{-1}
\end{array}\right]<0 \text { iv) }\left[\begin{array}{cc}
-\vartheta_{2} & \boldsymbol{e}^{T}(0) \\
* & -\boldsymbol{P}_{2}^{-1}
\end{array}\right]<0 \\
& \text { v) }\left[\begin{array}{cc}
-\boldsymbol{M}_{11} & \boldsymbol{N}_{11}^{T} \\
* & -\boldsymbol{Q}_{11}^{-1}
\end{array}\right]<0 \quad \text { vi) }\left[\begin{array}{cc}
-\boldsymbol{M}_{12} & \boldsymbol{N}_{12}^{T} \\
* & -\boldsymbol{Q}_{12}^{-1}
\end{array}\right]<0 \\
& \text { vii) } \left.\left[\begin{array}{cc}
-\boldsymbol{M}_{21} & \boldsymbol{N}_{21}^{T} \\
* & -\boldsymbol{Q}_{21}^{-1}
\end{array}\right]<0 \quad \text { viii }\right)\left[\begin{array}{cc}
-\boldsymbol{M}_{22} & \boldsymbol{N}_{22}^{T} \\
* & -\boldsymbol{Q}_{22}^{-1}
\end{array}\right]<0 \\
& \left.i x)\left[\begin{array}{cc}
-\boldsymbol{W}_{1} & \tau_{c 2} \boldsymbol{T}_{1}^{T} \\
* & -\tau_{c 2} \boldsymbol{R}_{1}^{-1}
\end{array}\right]<0 \quad x\right)\left[\begin{array}{cc}
-\boldsymbol{W}_{2} & \tau_{c 2} \boldsymbol{T}_{2}^{T} \\
* & -\tau_{c 2} \boldsymbol{R}_{2}^{-1}
\end{array}\right]<0 \\
& \left.x i)\left[\begin{array}{cc}
-\boldsymbol{G}_{1} & \tau_{s 2} \boldsymbol{S}_{1}^{T} \\
* & -\tau_{s 2} \boldsymbol{Z}_{1}^{-1}
\end{array}\right]<0 \quad x i i\right)\left[\begin{array}{cc}
-\boldsymbol{G}_{2} & \tau_{s 2} \boldsymbol{S}_{2}^{T} \\
* & -\tau_{s 2} \boldsymbol{Z}_{2}^{-1}
\end{array}\right]<0
\end{aligned}
$$

where:

$$
\begin{aligned}
& \int_{-\alpha_{1}}^{0} \boldsymbol{x}^{T}(s) \boldsymbol{x}(s) d s=\boldsymbol{N}_{11}^{T} \boldsymbol{N}_{11} \\
& \int_{-\alpha_{1}}^{0} \boldsymbol{e}^{T}(s) \boldsymbol{e}(s) d s=\boldsymbol{N}_{12}^{T} \boldsymbol{N}_{12} \\
& \int_{-\alpha_{2}}^{0} \boldsymbol{x}^{T}(s) \boldsymbol{x}(s) d s=\boldsymbol{N}_{21}^{T} \boldsymbol{N}_{21} \\
& \int_{-\alpha_{2}}^{0} \boldsymbol{e}^{T}(s) \boldsymbol{e}(s) d s=\boldsymbol{N}_{22}^{T} \boldsymbol{N}_{22}
\end{aligned}
$$

$\int_{-\tau_{c 2}}^{0} \int_{\theta}^{0} \dot{\boldsymbol{x}}^{T}(s) \dot{\boldsymbol{x}}(s) d s d \theta=\boldsymbol{T}_{1}^{T} \boldsymbol{T}_{1}$

$\int_{-\tau_{c 2}}^{0} \int_{\theta}^{0} \dot{\boldsymbol{e}}^{T}(s) \dot{\boldsymbol{e}}(s) d s d \boldsymbol{\theta}=\boldsymbol{T}_{2}^{T} \boldsymbol{T}_{2}$ 


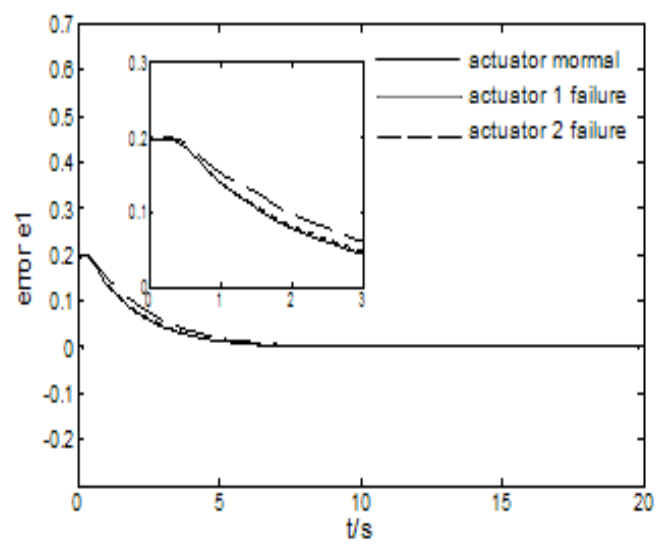

Fig. (2) response curve of the error vector.

The proposed optimization algorithm is a convex problem which is constrained by LMIs, and then it can be solved via the mincx solver of LMI tools.

\section{THE CIRCUIT}

In this section, we use an example to demonstrate the effectiveness of our main result.Consider the following NNCS with parameter uncertainties which is borrowed from [12]:

The membership function are $M_{1}\left(x_{2}\right)=\sin ^{2} x_{2}$ and $M_{2}\left(x_{2}\right)=\cos ^{2} x_{2}$

Rule 1: if $x 2$ is $M 1$, then

$\dot{\boldsymbol{x}}(t)=\left(\boldsymbol{A}_{1}+\Delta \boldsymbol{A}_{1}\right) \boldsymbol{x}(t)+\left(\boldsymbol{B}_{1}+\Delta \boldsymbol{B}_{1}\right) \boldsymbol{u}(t)$

$z(t)=C_{1} x(t)$

Rule 2: if $x 2$ is $M 2$, then

$\dot{\boldsymbol{x}}(t)=\left(\boldsymbol{A}_{2}+\Delta \boldsymbol{A}_{2}\right) \boldsymbol{x}(t)+\left(\boldsymbol{B}_{2}+\Delta \boldsymbol{B}_{2}\right) \boldsymbol{u}(t)$

$z(t)=C_{2} x(t)$

Where

$$
\begin{aligned}
& \boldsymbol{A}_{1}=\left[\begin{array}{cc}
-3 & 1 \\
1 & -1
\end{array}\right] \boldsymbol{A}_{2}=\left[\begin{array}{cc}
-2 & 1 \\
1 & 0
\end{array}\right] \boldsymbol{B}_{1}=\left[\begin{array}{cc}
1 & 0 \\
0 & -0.5
\end{array}\right] \\
& \boldsymbol{B}_{2}=\left[\begin{array}{cc}
1 & 0 \\
0 & 0.5
\end{array}\right] \boldsymbol{C}_{1}=\left[\begin{array}{cc}
1 & 0 \\
0 & -0.5
\end{array}\right] \boldsymbol{C}_{2}=\left[\begin{array}{cc}
1 & 0 \\
0 & 0.5
\end{array}\right]
\end{aligned}
$$

and matrices $\Delta \boldsymbol{A}_{i}$ and $\Delta \boldsymbol{B}_{i}(\mathrm{i}=1,2)$ satisfy $\left[\Delta \boldsymbol{A}_{i}, \quad \Delta \boldsymbol{B}_{i}\right]=$ $\boldsymbol{D F}(t)\left[\boldsymbol{E}_{a i}, \quad \boldsymbol{E}_{b i}\right]$, where

$$
\begin{aligned}
& \boldsymbol{D}=\left[\begin{array}{cc}
0.31 & 0.1 \\
0 & 0
\end{array}\right] \quad \boldsymbol{F}(t)=\left[\begin{array}{cc}
\sin t & 0 \\
0 & \cos t
\end{array}\right] \\
& \boldsymbol{E}_{a i}=\left[\begin{array}{cc}
0 & 0.2 \\
0 & 0
\end{array}\right] \quad \boldsymbol{E}_{b i}=\left[\begin{array}{cc}
0 & 0.2 \\
0 & 0
\end{array}\right] \quad(i=1,2)
\end{aligned}
$$

In cases of actuator normal and possible failures, the switching matrices $L_{0}=\operatorname{diag}(1,1), L_{1}=\operatorname{diag}(0,1)$ and $L_{2}=\operatorname{diag}$

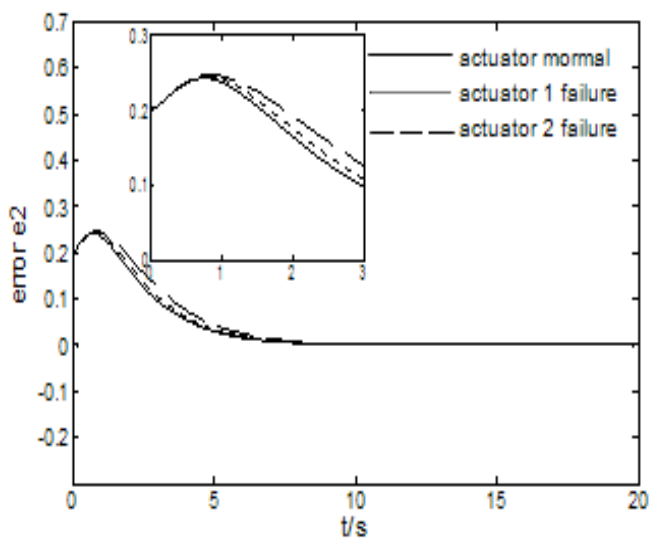

$(1,0)$ indicate actuator normal and actuator 1,2 failure, respectively.

Considering the actual NCS, we assume $T=0.05 \mathrm{~s}$, choose $d=0.4, \quad \delta_{i M}(i=1,2)=2, \quad \tau_{s c i}(i=1,2) \quad$ are $0.01 \mathrm{~s}$ and $0.1 \mathrm{~s}$, respectively. $\tau_{c a i}(i=1,2)$ are $0.01 \mathrm{~s}$ and $0.1 \mathrm{~s}$, respectively. Then we can solve convex problem (13), (14) by using LMI toolbox to obtain

$$
\begin{aligned}
& \boldsymbol{K}_{1}=\left[\begin{array}{ll}
-3.5235 & -0.3810 \\
1.2765 & -1.8105
\end{array}\right], \boldsymbol{K}_{2}=\left[\begin{array}{ll}
-4.2102 & -0.4680 \\
1.4688 & -1.9980
\end{array}\right] \\
& \boldsymbol{I}_{1}=\left[\begin{array}{ll}
-1.1745 & 0.1270 \\
0.4255 & 0.6035
\end{array}\right], \boldsymbol{I}_{2}=\left[\begin{array}{ll}
-1.1695 & 0.1300 \\
0.4080 & 0.5550
\end{array}\right]
\end{aligned}
$$

and guaranteed cost function $J \leq 32.246$.

Setting the initial conditions of the system $x(0)=[2,2]^{\mathrm{T}}$, $e(0)=[0.2,0.2]^{\mathrm{T}}$, in the cases of $L_{0}, L_{1}$ and $L_{2}$, response curve of the error vector $e 1, e 2$ are shown in Fig. (2), and zeroinput response curve of the state vector $x 1, x 2$ are shown in Fig. (3).

It can be seen from Fig. (2) that state estimation error quickly approximate to zero, which show that the states of closed-loop NNCS with possible actuator failures can be estimated well via the observer.

Fig. (3) can be seen that NNCFS is not only asymptotically stable, but also has good dynamic performance. These indicate that the proposed method makes the NNCS against possible actuator failures have the capability of robust faulttolerant guaranteed cost.

In addition, in terms of 3.2, we can receive the following optimal robust guaranteed cost fault-tolerant controller and observer gains:

$$
\boldsymbol{K}_{\text {opt } 1}=\left[\begin{array}{rr}
-0.7349 & -0.2540 \\
0.8521 & 1.2074
\end{array}\right] \quad \boldsymbol{K}_{\text {opt } 2}=\left[\begin{array}{rr}
-0.8339 & -0.2650 \\
0.8116 & 1.1210
\end{array}\right]
$$

the upper boundary of guaranteed cost function $J^{*}=28.363$.

\section{CURRENT AND FUTURE DEVELOPMENTS}

In this paper, based on T-S fuzzy model, we studies the control problem of the robust guaranteed cost fault-tolerant 

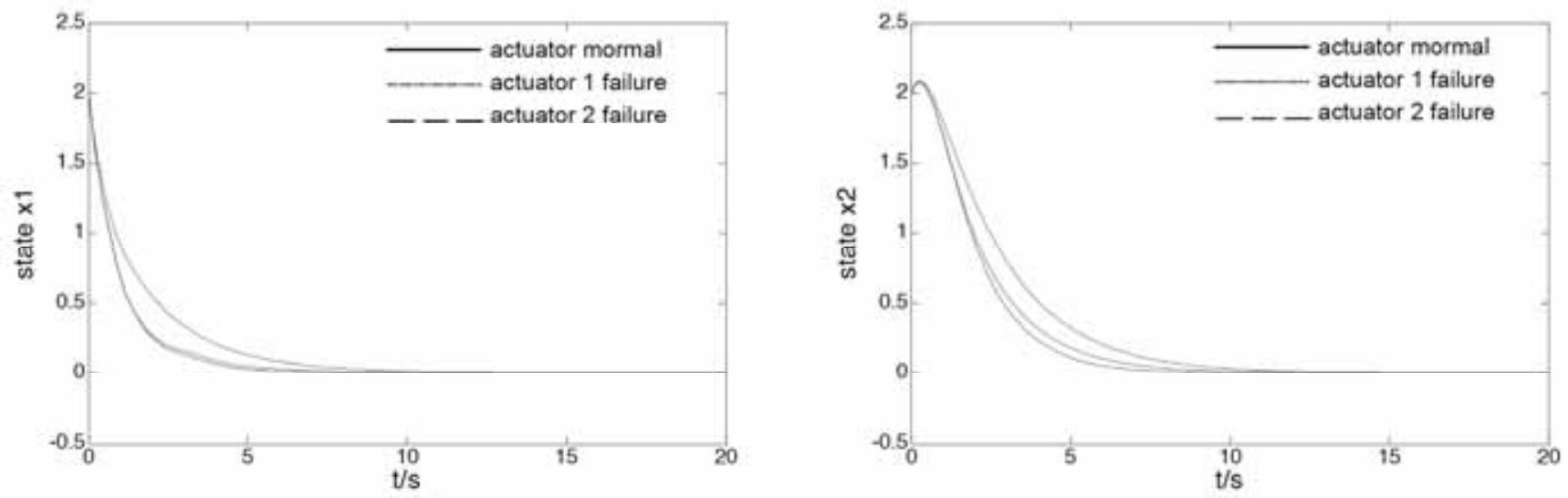

Fig. (3) response curve of the state vector.

on uncertain NNCS which includes time-delay and packet dropout under the condition of possible actuator failures. By constructing appropriate Lyapunov-Krasovskii functional, adopting the Jensen inequality and matrix separation technologies, this paper deduces a delay-dependent sufficient condition for robust guaranteed cost fault-tolerant. Further, we give the approach of solving for optimal controller and observer gains via optimizing. Finally, an example is used to illustrate the effectiveness and feasibility of proposed approach. In the future, in order to save the limited communication resources and improve the safety reliablity of NCS, we will research the active-passive hybrid fault-tolerant control under the event-trigger communication mechanism against the actuator failure.

\section{CONFLICT OF INTEREST}

The authors confirm that this article content has no conflict of interest.

\section{ACKNOWLEDGEMENTS}

This work is partially supported by National Natural Science Foundation of China under Grant 61364011, Gansu Natural Science Foundation under Grant 1212RJZA002.

\section{REFERENCES}

[1] B.G.W Lloyd, "Network control." European Patent 2075961, July 2009.

[2] M.S. Mcgee, M.S. Mcintyre, G.T. Howard, M.R. Enstone, and M.C. Stratton, "Network controller", U.S. Patent 7426189, September 2008.
[3] S. Hu and W.-Y. Yan, "Stability analysis of networked control systems with respect to packet loss", Automatica, vol. 43, no. 7, pp. 1243-1248, 2007.

[4] Y.J. Pan, H.J. Marquez and T. Chen, "Stabilization of remote control systems with unknown time varying delays by LMI techniques", Int. J. Control, vol. 79, no. 7, pp. 1668-1674, 2006.

[5] J. Li, Q. Zhang, H. Yu, and M. Cai, "Real-time guaranteed cost control of MIMO networked control systems with packet disordering", J. Process Contr., vol. 21, pp. 967-975, 2011.

[6] B.C. Wyld, J. Rosset, P. Sauvage, D. Pierrot, and D.M. Rondel, "Generic fault tolerant platform", U.S. Patent 5978933, November 1999.

[7] H. Jiandong, L. LI, and S. Sejun. "Fault-Tolerant Networking", European Patent EP1129563, December 2004.

[8] E.E. Solomon, "Fault-tolerant control system", U.S. Patent 4646065 , February 1987.

[9] R. J. Patton, C. Kambhampati, A. Casavola, and G. Franze, "Faulttolerance as a key requirement for the control of modern systems", The International Federation of Automatic Control, vol. 6, no. 1, pp. 26-36, 2006.

[10] H. Fang, H. Ye, and M. Zhong, "Fault diagnosis of networked control systems", Annu. Rev. Control, vol. 31, pp. 55-68, 2007.

[11] F. Jian amd W. Shen-Quan, "Reliable fuzzy control for a class of nonlinear networked control systems with time delay", Acta Automatica Sinica., vol. 38, no. 7, pp. 1091-1099, 2012.

[12] W. Jun, L. Wie, and L. Zhanming, "Design of robust Ho guaranteed cost and fault-tolerant for uncertain and nonlinear NCS", $J$. Huazhong Univ. Sci., vol. 39, no. 9, pp. 72-77, 2011. (in chinese)

[13] L. Wei, L. Wie, and J. Dong-nian, "Fault-tolerant control for nonlinear networked control system based on dynamic output feedback", J. Lanzhou Univ. Technol., vol. 38. no. 1, pp. 63-70, 2012. (in chinese)

[14] M. Takeuchi, "Fuzzy Controller", Japanese Patent JP 06035508. , February 1994.

[15] B. Barmish, "Necessary and sufficient conditions for quadratic stabilizability of an uncertain system", J. Optimiz. Theory App., vol. 46, no. 4, pp. 399-408, 1985.

[16] E. Tian, D. Yue, and C. Peng, "Quantized output feedback control for networked control systems", Inf. Sci., vol. 178, pp. 2734-2749, 2008.

[17] H. Gao, T. Chen, and J. Lam, "A new delay system approach to network-based control”, Automatica, vol. 44, pp. 39-52, 2008.

Received: June 09, 2014

(C) Wang et al.; Licensee Bentham Open.

This is an open access article licensed under the terms of the Creative Commons Attribution Non-Commercial License (http://creativecommons.org/licenses/by-nc/3.0/) which permits unrestricted, non-commercial use, distribution and reproduction in any medium, provided the work is properly cited. 